\title{
A ROBUST OPTIMIZATION APPROACH FOR INDEX TRACKING PROBLEM
}

\author{
${ }^{1}$ Mohsen Gharakhani, ${ }^{2}$ Forough Zarea Fazlelahi and ${ }^{3}$ S.J. Sadjadi \\ ${ }^{1}$ Department of Engineering, University of Qom, Qom, Iran \\ ${ }^{2}$ Department of Financial Engineering, University of Economic Sciences, Taleghani, Tehran, Iran \\ ${ }^{3}$ Department of Industrial Engineering, Iran University of Science and Technology, Narmak, Tehran, Iran
}

Received 2014-07-24; Revised 2014-08-28; Accepted 2014-09-24

\begin{abstract}
Index tracking is an investment approach where the primary objective is to keep portfolio return as close as possible to a target index without purchasing all index components. The main purpose is to minimize the tracking error between the returns of the selected portfolio and a benchmark. In this study, quadratic as well as linear models are presented for minimizing the tracking error. The uncertainty is considered in the input data using a tractable robust framework that controls the level of conservatism while maintaining linearity. The linearity of the proposed robust optimization models allows a simple implementation of an ordinary optimization software package to find the optimal robust solution. The proposed model of this study employs Morgan Stanley Capital International Index as the target index and the results are reported for six national indices including Japan, the USA, the UK, Germany, Switzerland and France. The performance of the proposed models is evaluated using several financial criteria e.g., information ratio, market ratio, Sharpe ratio and Treynor ratio. The preliminary results demonstrate that the proposed model lowers the amount of tracking error while raising values of portfolio performance measures.
\end{abstract}

Keywords: Robust Optimization, Index Tracking, Portfolio Selection, Mean Absolute Deviation Model, MinMax Model

\section{INTRODUCTION}

Fund management approaches can be divided into two categories: Active and passive management. In active management, the portfolio manager is willing to beat a predetermined index through trading stocks according to her experience and expertise. On the other hand, a passive manager tries to gain returns as close as possible to a theoretical portfolio e.g., the $\mathrm{S}$ and P 500 index. It is assumed that passive strategies can acquire higher returns in comparison to the active strategies in the long term. Index tracking is one of the passive portfolio management strategies which seeks to gain returns as close as possible to a target index without buying all index components.
There are typically two ways to find a portfolio that matches the performance of an index. In the first approach, called "full replication", the investor makes a portfolio including every constituent of the index proportional to its market share. In this approach, the investor could achieve a perfect match. However, incurring high transaction costs as well as the underlying complications for portfolio rebalancing make this approach inapplicable. The second approach, called "partial replication", includes investment in a small number of the stocks while attempting to imitate the performance of the entire index. This obviously incurs lower transaction costs and, at the same time, makes it easier to rebalance the portfolio weights when the market conditions change. Partial replication naturally allows Corresponding Author: Forough Zarea Fazlelahi, Department of Financial Engineering, University of Economic Sciences, Taleghani, Tehran, Iran, Tel: +61 414216533 
the investor to limit the choices of investment, for instance through insisting on the inclusion and/or exclusion of some pre-specified stocks by setting the proportion of the available capital that is to be invested. In fact, partial replication requires an error tracking measure to quantify the deviation of the tracking portfolio from the index.

Full replication has many drawbacks and augments the costs dramatically. Roll (1992), who solves the problem of minimizing tracking error, the variance of the difference between the returns of a benchmark and the target index, maximizes the average performance of a portfolio relative to a benchmark in a determined amount of tracking error volatility and considers a strategy for limiting beta, the systematic risk. Ammann and Zimmermann (2001) demonstrate the relation between the statistical measures of tracking error and asset allocation constraints and present a method for measuring them. Jansen and Dijk (2002) limit the number of stocks in a tracking portfolio and propose a new mathematical model. Jorion (2003) follows Roll (1992) by investigating the effect of a constraint on the tracking error volatility and interprets it as Value at Risk (VaR). He also portraits the methods for mitigating inefficiency of using constraints on TEVTracking Error Volatility- and shows the TEV-constrained portfolios by an ellipse in a mean-variance space.

Fabozzi et al., (2004) propose clustering as a methodology to construct a tracking portfolio. Gaivoronski and Pflug (2005) uses VaR as risk measure and introduces/sets forth mean-VaR frontiers. Konno et al. (2005) present a branch and bound algorithm for constructing or rebalancing a portfolio and use absolute deviations of returns rates. Yao et al. (2006) solve the index tracking problem with a portfolio containing only few assets. They formulate it as a stochastic quadratic control problem and solve the proposed model using semi-definite programming. Lai et al. (2006) present a Markowitzbased model for index tracking problem where they assume that index tracking problem relates to the constraining the probability of the tracking portfolio return falling below index return, generally referred to as downside risk. They presume that stock returns are jointly and normally distributed and that short selling is allowed which is not permissible in every stock market. Later, Bertrand (2010) following Jorion (2003), allows tracking error to vary instead of fixing tracking error volatility, TEV, but fixes risk aversion for volatility and explores iso-aversion frontiers.

The ultimate goal of the investor is to minimize the tracking error in her portfolio over time. Usually, tracking error is defined in a quadratic term. The resulting model can be expressed as a quadratic programming problem. The proportion of the entire capital to be invested in each stock is calculated as part of the same problem. Meade and Salkin (1990) formulate the portfolio selection problem as a quadratic program and investigate the effects of different policies used by fund managers on their returns.

Although quadratic programs have many interesting statistical properties, linear models are appealing from the computational aspect. Therefore, many attempts have been made to linearize the portfolio selection problem. Konno and Yamazaki (1991) proposes a portfolio optimization model that uses mean absolute deviation of the risk to deal with the modern portfolio theorysuggested by Markowitz (1952)-difficulties. Derigs and Nickel (2003) propose a simulated annealing-based metaheuristic for index tracking problem. In their approach, stock returns and covariances are derived from a linear multi-factor model based on macro-economic variables. They present a real-world case study based on an investment trust tracking, the German DAX30 index. They also consider their approach taking into account an investment portfolio of 500 available stocks in order to track the MSCI World Developed Market index.

Recently, there has been an extensive use of robust optimization in index tracking and other financial fields. Robust optimization technique can be used as a tool for handling uncertainty with data that was traditionally dealt with using sensitivity analysis, stochastic and fuzzy programming approaches. Robust optimization is a more tractable approach compared to the stochastic optimization that is especially suitable for problems in which input data and their corresponding distributions are uncertain (Gregory et al., 2011). The initiation of this technique is attributed to Soyster (1973) who proposes robust optimization models for over-conservative decision makers. Since then, other robust optimization approaches have been developed to address the issue with over-conservatism.

Robust optimization has been extensively applied in many financial applications (Fabozzi, 2007). Mulvey et al. (1995) develop a general robust optimization model and then compare it with different approaches of sensitivity analysis and stochastic linear programming. They solve some popular optimization problems like financial planning. Ben-Tal and Nemirovski (2002) propose a robust optimization methodology and apply it to some real world applications like portfolio selection. Goldfarb and Iyengar (2003) formulate robust portfolio selection problems. They systematically aim at combating the sensitivity of the optimal portfolio to statistical and modeling errors in the estimates of the 
relevant market parameters. El Ghaoui et al. (2003) propose a robust optimization approach for portfolio optimization problem with VaR. They define the worstcase $\mathrm{VaR}$ as the largest possible $\mathrm{VaR}$, given the partial information on the returns' distribution and consider the problem of computing and optimizing the worst-case $\mathrm{VaR}$ and show that these problems can be treated as semi-definite programs.

Bertsimas and Sim (2004) present a new approach for controlling excessive conservatism of solutions and call it "budget of uncertainty". Pinar and Tutuncu (2005) present a robust model in a multi-period setting and solve it as a quadratic programming problem. Kawas and Thiele (2011) suggest a new approach named log-robust in which they formulate the robust problem as a linear programming problem. Gregory et al. (2011) evaluate the cost of robustness for the robust counterpart to the maximum return portfolio optimization problem.

Further, in a review, Gabrel et al. (2013) present the areas in robust optimization that attracted the most attention in recent years. The most noticeable point in their paper is providing a big picture of robust optimization applications, particularly in finance. Nguyen and Lo (2012) apply a robust ranking model to portfolio optimization that develops a new ordering strategy for building a portfolio, instead of utilizing estimates of the parameters. Fertis et al. (2012) define the concept of robust risk measure as the worst possible of risks when each of probability measures are feasible. They also present a robust $\mathrm{CVaR}$ that is optimized by convex duality methods. Doan et al. (2013) present a distributional robust portfolio optimization model depending on overlapping multivariate marginal distribution information that can be solved by linear programming. Zhu et al. (2013) propose a portfolio selection framework based on dual robustness with a mixture distribution on returns. They show that their proposed model can be reformulated as either linear or second-order cone program.

To the best of our knowledge, Chen and Kwon (2012) are the only practitioners to investigate index tracking with robust approach. They present a model using a 0-1 integer program in which they allow uncertainty in the objective function. Their tractable robust framework controls conservatism of the solution which protects against worst-case realizations of potential estimation errors and other deviations.

Rudolf et al. (1999) propose four linear models for minimizing the tracking error between the returns of a portfolio and a benchmark. Their idea of linearization is based on the fact that in the real market we confront with linear performance fees of fund managers; therefore, they argue that linear deviations give a more accurate description of the investors risk attitude rather than squared deviations. The main common feature within all models is that absolute deviations are minimized instead of squared deviations as is the case for traditional optimization models. In order to compare the performance of different approaches, they adopt a real data set containing six national stock market indices, including the USA, Japan, the UK, Germany, France and Switzerland and the tracking error with respect to the MSCI (Morgan Stanley Capital International Index) world stock market index is minimized. They also show that linear tracking error optimization is equivalent to expected utility maximization and lower partial moment minimization. This study is closely related to the last work of Rudolf et al. (1999). We propose a robust optimization approach and compare different models using recent data sets (2003-2011). In this study, four models based on the mean absolute deviation are presented and their robust counterparts are derived. The proposed robust models have linear forms as in Bertsimas and Sim (2004) which makes them computationally tractable.

The organization of this study is as follows. Section 2 presents different models and formulates the well-known quadratic as well as the linear models. Robust counterparts of some linear programming models are discussed in section 3. Section 4 compares the performance of different models using a real-world case study based on a broad spectrum of performance criteria. Finally, concluding remarks are presented in section 5 to put in a nutshell the contribution of this study.

\section{QUADRATIC AND LINEAR MODELS}

In this section, we formulate a quadratic model and four linear models including Mean Absolute Deviation (MAD), Mean Absolute Downside Deviation (MADD), MinMax Deviation (MinMax) and Downside MinMax Deviation (DMinMax) for tackling tracking error in passive portfolio management. The ultimate objective of the investor is to find the portfolio weights that follow the benchmark return as close as possible. This close tracking of index return is defined based on a variety of distance measures.

\subsection{Model Formulations}

Consider an investor who chooses $n$ assets of the index to construct the tracking portfolio. Let $\beta(n \times 1$ vector) represent the portfolio weights, $Y(T \times 1$ vector) be the vector of market index return over time and $X(T \times n$ matrix) be the matrix of returns on $n$ assets for each time 
period where the returns are continuously compounded. $T$ is the number of observations and $\varepsilon$ is the deviation between returns of the tracking portfolio and the target index. In this formulation, $X \beta$ denotes the tracking portfolio return. Using the distance definitions to measure the error, the index tracking problem could be formulated as in the following sections.

\subsubsection{Mean Square Formulation}

Similar to Rudolf et al. (1999) the objective function is to minimize some function of tracking error over stock weights, $\beta$. Typically, the deviation between the tracking portfolio and the index is defined as Equation 1:

$\varepsilon=Y-X \beta, Y \in R^{T}, X \in R^{(T \times n)}, \beta \in R^{n}, \varepsilon \in R^{T}$

Therefore, the mean square tracking error based on Roll (1992) is presented as Equation 2:

$$
\min _{\beta} \varepsilon^{\prime} \varepsilon \equiv \min _{\beta}(Y-X \beta)^{\prime}(Y-X \beta)
$$

It can be verified that the vector of optimum asset weights in the mean square tracking error of Equation (2) is $\beta=\left(X^{\prime} X\right)^{-1} X^{\prime} Y$. We can add some linear constraints such as short-selling that requires positivity of the portfolio weights. Furthermore, the sum of the weights is equal to one, which means the sum of asset weights within the portfolio must add up to unity. Therefore, the mean square tracking error problem can be formulated as the following quadratic programming problem Equation 3:

$$
\begin{aligned}
& \min _{\beta}(Y-X \beta)^{\prime}(Y-X \beta) \\
& \text { s.t.: } \\
& 1^{\prime} . \beta=1, \text { where } 1^{\prime} \equiv(1, \ldots, 1) \in R^{n} \\
& \beta \geq 0, \text { where } 0 \equiv(0, . ., 0) \in R^{n}
\end{aligned}
$$

\subsubsection{Linear Models for the Tracking Error Minimization Problem}

The linear models presented here minimize absolute deviations between the targeted index and the tracking portfolio returns. The first linear model is called MAD, which minimizes the average of absolute deviations. The second model, called MinMax, minimizes the maximum deviation between returns of tracking portfolio and the benchmark. Another approach is to focus only on the negative deviations of tracking portfolio from the benchmark returns. The reason is that from the investor's point of view, risk happens only when the return of the tracking portfolio falls below the benchmark, known as "downside risk" of an investment (Harlow, 1991). The objective function of different models is summarized as follows Equation $4 \mathrm{a}$ and $\mathrm{b}$ :

$$
\begin{aligned}
& T E_{Q D}=\min _{\beta}(Y-X \beta)^{\prime}(Y-X \beta) \\
& T E_{M A D}=\min _{\beta} 1^{\prime}(|X \beta-Y|), \\
& T E_{M A D D}=\min _{\beta} 1^{\prime}(|\bar{X} \beta-\bar{Y}|),
\end{aligned}
$$

$$
\text { for some } t \text { where } \bar{X}_{t} \beta<\bar{Y}_{t} \text {, }
$$

$T E_{\text {MinMax }}=\min _{\beta}\left\{\max _{t}|X \beta-Y|\right\}$

$T E_{D M i n M a x}=\min _{\beta}\left\{\max _{t}|\bar{X} \beta-\bar{Y}|\right\}$,

for some $t$ where $\bar{X}_{t} \beta<\bar{Y}_{t}$,

where, $X_{t}$ represents row $t$ of matrix $X$ and $Y_{t}$ is the element $t$ th of vector $Y$, while matrices $\bar{X}$ and vector $\bar{Y}$ consider only those rows $t$ where $\bar{X} \beta<\bar{Y}$.

In order to derive the MinMax models in the classical linear form, we define an auxiliary variable $z \geq 0$ as the upper bound of absolute deviations similar to Rudolf et al. (1999). Hence, for the MinMax model we have:

$$
z \geq\left|X_{t} \beta-Y_{t}\right|, t=1, \ldots, T
$$

Two forms can be considered for each $t$ :

$$
\begin{aligned}
& \text { if } X_{t} \beta \geq Y_{t} \Leftrightarrow X_{t} \beta-z \leq Y_{t} \\
& \text { if } X_{t} \beta \leq Y_{t} \Leftrightarrow X_{t} \beta+z \geq Y_{t}
\end{aligned}
$$

Thus, we can formulate the MinMax model as follow Equation 5:

$$
\begin{aligned}
& \min _{z} z \\
& \text { s.t. } X_{t} \beta-z \leq Y_{t} \\
& X_{t} \beta+z \geq Y_{t}
\end{aligned}
$$


Since in the $D$ MinMax model only the positive deviations of index is of interest, we derive Equation 6:

$\min _{z}$ zs.t. $X_{t} \beta+z \geq Y_{t}$.

Let $z_{t}^{+}$and $z_{t}^{-}$represent the absolute value of positive and negative deviations of tracking portfolio at time period $t$, respectively. The MAD model can be formulated as Equation 7:

$$
\begin{aligned}
& \operatorname{Min} \sum_{t=1}^{T}\left(z_{t}^{+}+z_{t}^{-}\right) \\
& \text {s.t } X_{t} \beta-z_{t}^{+}+z_{t}^{-}=Y_{t}
\end{aligned}
$$

For the MADD model, we omit $z_{t}^{+}$from the objective function of problem (7). Then, the problem is stated as Equation 8:

$$
\begin{aligned}
& \min \sum_{t=1}^{T} z_{t}^{-} \\
& \text {s.t. } X_{t} \beta-z_{t}^{+}+z_{t}^{-}=Y_{t} .
\end{aligned}
$$

\section{ROBUST FORMULATION}

In the classic linear programming, we typically assume that the input data is precisely known and their values are known with certainty. However, in the real world optimization problems this assumption does not hold since it ignores underlying effects of parameter uncertainty in the model. This could potentially affect the underlying feasibility and/or optimality of the final solution. After/when the model is solved using the nominal values (i.e., the expected value of data), future realizations of the parameters may violate some constraints and therefore, the so-called optimal solution which is based on the nominal data may no longer even exist in the feasible region.

In order to overcome the above-mentioned drawbacks, robust optimization approach is used in the real-world applications of linear programming (Bertsimas and Sim, 2004). Soyester (1973) proposes a conservative technique where the solution remains feasible for all realizations of input data. He considers the worst-case solution where the uncertain parameters belong to a convex set. There are some other approaches in the literature that try to address the over conservatism of the Soyester approach. Other researchers including El-Ghaoui et al. (1998), Ben-Tal and Nemirowski $(1998,1999,2000)$ and Bertsimas and Sim (2004) address the uncertainty of data using different robust approaches. The distinction among these approaches is the way they define uncertainty sets. They solve a new formulation of the original problem called the robust counterpart. The robust counterpart proposed by BenTal and Nemirowski $(1998,1999,2000)$ is typically in the form of conic quadratic programming since they apply elliptic uncertainty. Bertsimas and Sim (2004) propose a new approach wherein they define a linear norm and a linear uncertainty set. Their approach preserves the linearity of the original problem, which is computationally efficient. Consider the following simple linear programming where the technical matrix is subject to uncertainty Equation 9:

$$
\begin{aligned}
& \max c^{\prime} x \\
& \text { s.t.: } \\
& \tilde{A} x \leq b \\
& l_{j} \leq x_{j} \leq u_{j} \forall j
\end{aligned}
$$

where, data uncertainty only affects the elements in technical matrix $\tilde{A}$. Furthermore, assume- without loss of generality-that the objective function $c^{\prime} x$ is not subjected to uncertainty, since in that case the objective function can be converted into a simple constraint and be added to the main constraints.

They propose another linear programming problem called the robust counterpart, which could be solved instead of the nominal problem (9) as follows Equation 10:

$$
\begin{aligned}
& \max ^{\prime} x \\
& \text { s.t.: } \\
& \sum_{j} a_{i j} x_{j}+z_{i} \Gamma_{i}+\sum_{j} p_{i j} \leq b_{i}, \forall i \\
& z_{i}+p_{i j} \geq \hat{a}_{i j} y_{j}, \forall i, j \in J_{i}, \\
& -y_{j} \leq x_{j} \leq y_{j}, \forall j, \\
& l_{j} \leq x_{j} \leq u_{j}, \forall j, \\
& p_{i j} \geq 0, \forall i, j \in J_{i}, \\
& y_{j} \geq 0, \forall j, \\
& z_{i} \geq 0, \forall i .
\end{aligned}
$$

where, $a_{i j}$ is the nominal value of uncertain parameter $\tilde{a}_{i j}, \hat{a}_{i j}$ is the allowed deviation from the nominal value, i.e., we consider $\tilde{a}_{i j}$ takes values in $\left[a_{i j}-\hat{a}_{i j}, a_{i j}+\hat{a}_{i j}\right]$. Let $J_{i}$ define the set of coefficient (s) in constraint $i$ that may be subjected to uncertainty. The parameter $\Gamma_{i}$, taking value in $\left[0, J_{i}\right]$, is defined for each constraint $i$ and is not necessarily integer. This parameter controls the robustness of the solution against the level of conservatism, e.g., for $\Gamma_{i}=0$ the robust counterpart is equivalent to the nominal problem and on the other hand 
$\Gamma_{i}=J_{i}$ represents the worst-case situation and is equivalent to the Soyster approach. In the above model, $y_{j}$ is defined as absolute value of $\mathrm{x}_{\mathrm{j}} \cdot z_{i}$ and $p_{\mathrm{ij}}$ are some auxiliary variables that together define the protection function of constraint $i$ (Bertsimas and Sim, 2004).

In the robust optimization we are not allowed to keep equality constraint in the model, so the robust counterpart for the MAD and MADD cannot be defined. In this study, we formulate the robust counterpart of both MinMax and Downside MinMax problem based on Bertsimas and Sim (2004) approach. In this case, consider $\beta$ as the portfolio weights and $\tilde{X}_{t j}$ as uncertain return of stock $i$ at time period $t$. We assume the uncertain return $X_{t j}$ takes value within interval $\left[\hat{x}_{t j}-\Delta, \hat{x}_{t j}+\Delta\right]$, where $\Delta$ is the deviation parameter. Based on the assumptions and definitions mentioned above we formulate the robust counterpart of MinMax (RMinMax afterwards) and DMinMax (RDMinMax afterwards) problems as a similar approach.

The RMinMax model is formulated as Equation 11:

$$
\min z
$$

s.t.:

$\sum_{j=1}^{N} \hat{x}_{t j} \beta_{j}-z+W_{t} \Gamma_{t}+\sum_{j=1}^{N} P_{t j} \leq y_{t}$

$W_{t}+P_{t j} \geq \Delta \hat{x}_{t j} u_{j}, \forall t, j$

$-u_{j} \leq \beta_{j} \leq u_{j}, \forall j$

$P_{t j} \geq 0, \forall t, j$

$W_{t} \geq 0, \forall t$

$u_{j} \geq 0, \forall j$

The RDMinMax model is formulated as Equation 12:

$$
\begin{aligned}
& \min z \\
& \text { s.t.: } \\
& \sum_{j=1}^{N} \hat{x}_{t j} \beta_{j}+z-W_{t} \Gamma_{t}-\sum_{j=1}^{N} P_{t j} \geq y_{t} \\
& W_{t}+P_{t j} \geq \Delta \hat{x}_{t j} u_{j}, \forall t, j \\
& -u_{j} \leq \beta_{j} \leq u_{j}, \forall j \\
& P_{t j} \geq 0, \forall t, j \\
& W_{t} \geq 0, \forall t \\
& u_{j} \geq 0, \forall j
\end{aligned}
$$

In all proposed models, short-selling is prohibited and finally, there is a constraint on portfolio weights that must add up to unity.

\section{EXPERIMENTAL RESULTS}

In this section, we implement previously proposed models using a real data set to compare their performance empirically. Consider an investor who is interested in constructing an internationally diversified portfolio. Additionally, she has an objective to minimize the tracking error between her portfolio return and the world stock index. MSCI index, which measures the performance of equity market within developed countries, is used as the target index. The index has been maintained by Morgan Stanley Capital International incorporation since 1969. MSCI world constitutes of indices of 24 developed country market indices, which is a common benchmark for the global equity funds. It should be noted that emerging markets index has been excluded from the benchmark.

The investor is going to compose a portfolio of market index of 6 industrial countries: The United States (the USA), Germany (D), France (F), the United Kingdom (the $\mathrm{UK})$, Switzerland (CH) and Japan (JAP). The objective is to minimize the tracking error between the returns of the composed portfolio and the MSCI world index. Monthly data, including 100 monthly return observations, are collected from January 2003 to September 2012. Moreover, the whole observation period is divided into two sub-periods: 80 Observations used as train sample observations from January 2003 to August 2009 and 20 observations from September 2009 to September 2012 are assumed as test sample observations. Train period data are used for constructing optimized portfolios for the obtained models. Then, the test sample observations are used to test the efficiency of obtained portfolio weights. All returns are calculated in USD. Mean, standard deviation and $\beta$ relative to the MSCI world index of each series of data are summarized in Table 1.

The proposed modeling is solved using Lingo software. Table 2 contains the weights of optimized portfolios for linear and robust models. In addition, values of objective functions are calculated for each model in a separate column.

Bertsimas and Sim (2004) propose some probability bounds of constraint violation. Based on their propositions, we set the parameter $\Gamma$ and the budget of uncertainty to limit the upper bound for the probability of violation for each constraint up to $5 \%$. It is noteworthy that the parameter $\Gamma$ controls the underlying trade-off between the probability of violation and the effect of the objective function of the nominal problem. We tuned the robust model parameter in such a way that the constraint would not to be violated with $95 \%$ confidence interest. 
Table 1. Risk/return characteristics of MSCI total return indices in terms of USD

\begin{tabular}{|c|c|c|c|c|c|c|c|c|c|}
\hline \multirow[b]{2}{*}{ Index } & \multicolumn{3}{|c|}{$\begin{array}{l}\text { Whole observation period: } \\
\text { Jan } 2003 \text { to Sep } 2012\end{array}$} & \multicolumn{3}{|c|}{$\begin{array}{l}\text { Train sample period: } \\
\text { Jan } 2003 \text { to Aug } 2009\end{array}$} & \multicolumn{3}{|c|}{$\begin{array}{l}\text { Test sample period: } \\
\text { Sep } 2009 \text { to Sep } 2012\end{array}$} \\
\hline & $\mu$ & $\sigma$ & $\beta$ & $\mu$ & $\Sigma$ & $\beta$ & $\mu$ & $\Sigma$ & $\beta$ \\
\hline MSCI & 0.44 & 4.79 & 1.00 & 0.27 & 4.81 & 1.00 & 1.12 & 4.76 & 1.00 \\
\hline USA & 0.36 & 4.41 & 0.90 & 0.10 & 4.37 & 0.89 & 1.38 & 4.51 & 0.92 \\
\hline JAP & 0.27 & 5.00 & 0.77 & 0.33 & 5.20 & 0.82 & -0.01 & 4.20 & 0.59 \\
\hline UK & 0.31 & 5.31 & 1.04 & 0.14 & 5.28 & 1.03 & 1.01 & 5.48 & 1.09 \\
\hline D & 0.77 & 7.11 & 1.39 & 0.63 & 7.21 & 1.40 & 1.31 & 6.87 & 1.35 \\
\hline $\mathrm{F}$ & 0.46 & 6.51 & 1.29 & 0.42 & 6.22 & 1.24 & 0.60 & 7.75 & 1.52 \\
\hline $\mathrm{CH}$ & 0.71 & 4.82 & 0.87 & 0.59 & 4.84 & 0.89 & 1.15 & 4.82 & 0.78 \\
\hline
\end{tabular}

Monthly average return and standard deviation are in \%.

$\beta$ is respect to MSCI.

Table 2. Optimized portfolio weights based on the train period (Jan 2003 to Aug 2009) for different optimization models

\begin{tabular}{lllllrrr}
\hline Model & USA & JAP & UK & D & \multicolumn{1}{r}{ F } & CH & Value of objective function \\
\hline QTE & 50.28 & 11.80 & 18.63 & 6.43 & 12.87 & 0.00 & 2.55 \\
MAD & 51.46 & 11.12 & 20.54 & 7.13 & 9.75 & 0.00 & 17.49 \\
MADD & 48.70 & 11.80 & 17.63 & 9.36 & 11.66 & 0.84 & 10.25 \\
MinMax & 49.84 & 12.57 & 14.50 & 7.64 & 15.45 & 0.00 & 0.69 \\
DMinMax & 49.06 & 11.77 & 19.29 & 14.07 & 5.81 & 0.00 & 0.59 \\
RMinMax & 22.20 & 15.34 & 23.78 & 12.89 & 12.89 & 12.89 & 8.81 \\
RDMinMax & 26.23 & 11.85 & 26.36 & 11.85 & 11.85 & 11.85 & 8.80 \\
\hline
\end{tabular}

Square root of objective function is calculated for QTE.

All figures in percent $\%$.

Clearly, high weights are dedicated to the USA, the UK and JAP stock markets. There is an obvious result due to the fact that MSCI index is capital weighted and these countries have the highest capital values of market index in the world. It is worth noting that MAD, MADD and quadratic tracking error results are similar in a noteworthy manner. This result is consistent with the perceptions of Konno and Yamazaki (1991) and signifies that the optimization results are the same whether linear or quadratic objective functions are used when the joint probability distribution of the benchmark and portfolio returns is normally distributed. Moreover, Rudolf et al. (1999) report the same results in their empirical work. Slight differences could be expected between the MAD and the quadratic tracking error model since the normality of the returns is non-existent. Moreover, MAD and the MinMax models have almost different results, which is not surprising due to the differences among the tracking error criteria. It could be observed that the portfolio weights differ substantially between the robust and non-robust models (Table 2). For instance, the weight of the US market in the portfolio declines to almost half of its value when we use the robust models instead of the nominal models. The most significant feature of the robust models is that the portfolio weights are distributed in a more uniform and diversified manner/uniformity and diversity of the portfolio weights distribution (Table 2).
So far, the results have disclosed that the linear models provide quite different portfolios than the traditional quadratic models. The benefit of the linear models, however, is that the value of the objective function provides an intuitive and immediate interpretation. It is easier for an investor to determine her attitude toward the concept of risking if she can express the tracking error in terms of absolute deviations from the benchmark index rather than squared deviations. A comparison of the optimized values of the objective functions is summarized in Table 3 .

The minimum values of the objective function across the different models are in bold face. For example, the lowest tracking error of a portfolio regarding the benchmark using the quadratic model is provided by the QTE portfolio. Nevertheless, more interesting insights emerge from the objective functions of the alternative models. For instance, if an investor is concerned about the maximum absolute downside deviation (DMinMax), the maximum risk she takes by holding the Downside MinMax portfolio is $0.588 \%$. This is quite lower than the QTE portfolio which has a maximum Downside deviation (DMinMax) of $0.759 \%$ from the benchmark. If the investment objective is the Minimum sum of Absolute Downside Deviations (MADD), the total deviation may be boosted by $3.85 \%$ in case the QTE portfolio is substituted by the MADD portfolio. 
Table 3. Optimal objective function values for different optimization models over the train period (Jan 2003 to Aug 2009) Tracking error criteria

\begin{tabular}{|c|c|c|c|c|c|}
\hline Model & QTE & MAD & MADD & MinMax & DMinMax \\
\hline QTE & 0.065 & 17.671 & 10.662 & 0.820 & 0.759 \\
\hline MAD & 0.068 & 17.490 & 10.909 & 0.908 & 0.770 \\
\hline MADD & 0.073 & 18.363 & 10.251 & 0.793 & 0.669 \\
\hline MinMax & 0.076 & 19.095 & 10.655 & 0.685 & 0.685 \\
\hline DMinMax & 0.085 & 19.734 & 10.722 & 1.014 & 0.588 \\
\hline RMinMax & 0.375 & 44.760 & 19.223 & 1.353 & 1.353 \\
\hline RDMinMax & 0.304 & 40.712 & 18.165 & 1.484 & 1.133 \\
\hline
\end{tabular}

All figures in $\%$.

It is obvious that robust model objectives are not minimum values in any criteria. For example, in some cases, the tracking error criteria have grown twofold. However, it should not be considered as the pitfalls of the robust modeling. The minimum possible value could be gained since the objective function of each model optimizes the weight. We cannot compare these models using the same data, applied to find tracking portfolio, i.e., the train data. To the best of the investor's behavior, the investor definitely knows that this would deteriorate the objective function when robust modeling is used. The investor trades return for confidence. Using the test data set, the performance of different portfolio is tested in the following section.

\subsection{Performance Metrics}

In this section, the most important portfolio measures are summarized in Table $\mathbf{4}$ according to which we could examine the performance of different models. It is apparent that the higher these measures, the better the performance of the portfolio. We also test their performance using test sample returns so that we can arrive at an unbiased judgment.

In this table, $R_{i}$ and $R_{b}$ are the returns of tracking portfolio and target benchmark index, respectively. $T E$ is the tracking error and $\sigma$ is the standard deviation of the portfolio return.

\subsection{Numerical Results of Performance Criteria}

In this section, we compare different portfolio models based on multiple investment performance criteria. In Table 5, average returns, standard deviations, tracking errors and different market-related ratios for the portfolios are displayed. Given the previous results, it is astounding how similar the risk characteristics are. Except for the MAD and the quadratic QTE model, average returns are considerably different. The well-known observation is that the higher the expected return, the higher the standard deviation. The Information, Sharp and Treynor ratios of the non-robust models are negative, which represent an inferior performance with respect to market index. However, the robust models have higher returns together with positive market-related ratios that reveal the superior performance regarding non-robust approaches to the market portfolio. A graphical comparison of different MinMax models together with their robust counterparts is illustrated in a radar diagram (Fig. 1).

The relevant data set is normalized to depict Fig. 1. It could be observed that regarding train period returns, robust models outperform non-robust models based on market-related portfolio performance measures.

\subsection{Further Analysis Applying Test Data}

So far, the comparisons were conducted based on the train data, i.e., the data used to obtain optimized portfolio of each tracking error approach. In this section, the test data are used in order to test viability of the results. Keeping in mind that the data set used for testing the results (test data; the period from September 2009 to September 2012) is a very extraordinary circumstance wherein a huge financial crisis occurs and the world witnesses lots of important economic and political changes affecting the stock markets. In Table 6, average returns, standard deviations, tracking errors and different market-related ratios for the portfolios are displayed.

An outstanding outcome is that Beta of different models are all negative; this proves that the returns are moving in the opposite direction of the index in order to get higher returns.

Figure 2 depicts the tracking error of robust models with respect to the world index fluctuations.

It can be seen that the resulting error is directly related to the world index. Table 7 summarizes monthly returns of each portfolio in each column and the world index monthly returns in the last column. One could report that the returns are fluctuating in the same way with world return when comparing different methods in almost all observation period. 
In order to increase validity of our comparison, we added noise to our test data to simulate data for 50 runs. We added a random noise to the test data that has normal distribution with mean and standard deviation equal to 0 and 0.2 , respectively. Then, we ran the simulation for 50 times. The calculated tracking error values for the noisy data are given in Table 8. It is noticeable that the tracking error values for robust models are less than the tracking error values for the non-robust models. This suggests that the robust models have a better performance for the noisy data. Figure 3 compares the relative tracking error of different models for noisy data with respect to nominal test data for 50 runs of simulation. The robust models have lower amount of relative tracking error among models. This occurs because when the noise is added to data, robust models are expected to change less in their objective functions with respect to non-robust models.

Table 4. Performance metrics formulations and descriptions

\begin{tabular}{|c|c|c|}
\hline Performance metric & Formulation & Description \\
\hline Beta & $\beta=\frac{\operatorname{cov}\left(R_{i}, R_{b}\right)}{\operatorname{var}\left(R_{i}\right)}$ & Beta of a portfolio is a number which shows he relationship between the return \\
\hline Information ratio $^{\mathrm{a}}$ & $I R=\frac{E\left[R_{i}-R_{b}\right]}{\sqrt{T E}}$ & $\begin{array}{l}\text { of the portfolio and a benchmark } \\
\text { Information ratio, typically known as Appraisal Ratio, is the difference between }\end{array}$ \\
\hline & & $\begin{array}{l}\text { returns of tracking portfolio and a selected benchmark divided by the standard } \\
\text { deviation of the tracking error }\end{array}$ \\
\hline Sharpe ratio ${ }^{b}$ & $S=\frac{E\left[R_{i}-R_{b}\right]}{\sigma}$ & Sharpe ratio is a measure of excess return of a trading strategy or tracking \\
\hline & & portfolio for each unit of risk the investor takes \\
\hline Treynor ratio ${ }^{c}$ & $T=\frac{E\left[R_{i}-R_{b}\right]}{\beta_{i}}$ & Treynor ratio measures the returns obtained above what the investment could gain \\
\hline Market ratio $^{\mathrm{d}}$ & $M=\frac{1+R_{i}}{1+R_{b}}$ & Market ratio measures the relative performance of tracking portfolio to benchmark index \\
\hline
\end{tabular}

${ }^{\mathrm{a}}$ Sharpe (1994)

${ }^{\mathrm{b}}$ Sharpe 1966)

${ }^{\mathrm{c}}$ Treynor (1964)

${ }^{\mathrm{d} C}$ Cornuejols and Tutuncu (2007)

Table 5. Risk/return characteristics of optimized portfolios based on train period (Jan 2003 to Aug 2009)

\begin{tabular}{lllllllll}
\hline Model & $\begin{array}{l}\text { Average } \\
\text { return }^{\mathrm{a}}\end{array}$ & $\begin{array}{l}\text { Standard } \\
\text { deviation }^{\mathrm{a}}\end{array}$ & $\begin{array}{l}\text { Tracking } \\
\text { error }^{\mathrm{b}}\end{array}$ & Beta $^{\mathrm{c}}$ & $\begin{array}{l}\text { Information } \\
\text { ratio }^{\mathrm{d}}\end{array}$ & $\begin{array}{l}\text { Sharpe } \\
\text { ratio }^{\mathrm{e}}\end{array}$ & $\begin{array}{l}\text { Treynor } \\
\text { ratio }^{\mathrm{f}}\end{array}$ & $\begin{array}{l}\text { Market } \\
\text { ratio }^{\mathrm{g}}\end{array}$ \\
\hline QTE & 0.232 & 4.735 & $2.83 \mathrm{E}-03$ & 0.9830 & -0.1467 & $-8.78 \mathrm{E}-03$ & $-4.23 \mathrm{E}-04$ & 0.9996 \\
MAD & 0.225 & 4.715 & $2.89 \mathrm{E}-03$ & 0.9788 & -0.1696 & $-1.04 \mathrm{E}-02$ & $-5.00 \mathrm{E}-04$ & 0.9995 \\
MADD & 0.249 & 4.782 & $3.02 \mathrm{E}-03$ & 0.9924 & -0.0824 & $-5.21 \mathrm{E}-03$ & $-2.51 \mathrm{E}-04$ & 0.9998 \\
MinMax & 0.248 & 4.780 & $3.08 \mathrm{E}-03$ & 0.9919 & -0.0838 & $-5.40 \mathrm{E}-03$ & $-2.60 \mathrm{E}-04$ & 0.9997 \\
DMinMax & 0.253 & 4.813 & $3.27 \mathrm{E}-03$ & 0.9984 & -0.0643 & $-4.37 \mathrm{E}-03$ & $-2.10 \mathrm{E}-04$ & 0.9998 \\
RMinMax & 0.346 & 4.963 & $6.85 \mathrm{E}-03$ & 1.0223 & 0.1053 & $1.45 \mathrm{E}-02$ & $7.05 \mathrm{E}-04$ & 1.0007 \\
RDMinMax & 0.323 & 4.940 & $6.18 \mathrm{E}-03$ & 1.0192 & 0.0795 & $9.94 \mathrm{E}-03$ & $4.82 \mathrm{E}-04$ & 1.0005 \\
\hline
\end{tabular}

\footnotetext{
${ }^{\mathrm{a}}$ Monthly returns and standard deviations in \%

${ }^{\mathrm{b}}$ Tracking error measured by standard deviation of portfolio excess return to MSCI ( $\left.\sigma(\mathrm{XB}-\mathrm{Y})\right)$

c eta of each portfolio to MSCI world stock market index

${ }^{\mathrm{d}}$ Excess return to square root of tracking error in \%

${ }^{\mathrm{e}}$ Excess return to volatility ratio in $\%$

${ }^{\mathrm{f}}$ Excess return to Beta in \%

${ }^{g}$ Relative performance of tracking portfolio to benchmark index
} 
Mohsen Gharakhani et al. / Journal of Computer Science 10 (12): 2450.2463, 2014

Table 6. Risk/return characteristics of optimized portfolios based on test sample period (Sept 2009 to Sep 2012)

\begin{tabular}{|c|c|c|c|c|c|c|c|c|}
\hline Model & $\begin{array}{l}\text { Average } \\
\text { return }\end{array}$ & $\begin{array}{l}\text { Standard } \\
\text { Deviation }^{\mathrm{a}}\end{array}$ & $\begin{array}{l}\text { Tracking } \\
\text { Error }^{\mathrm{b}}\end{array}$ & Beta $^{c}$ & $\begin{array}{l}\text { Information } \\
\text { ratio }^{\mathrm{d}}\end{array}$ & $\begin{array}{l}\text { Sharpe } \\
\text { ratio }\end{array}$ & $\begin{array}{l}\text { Treynor } \\
\text { ratio }\end{array}$ & $\begin{array}{l}\text { Market } \\
\text { ratiog }\end{array}$ \\
\hline QTE & 1.069 & 4.855 & $3.144 \mathrm{E}-03$ & 1.0173 & -0.1695 & $-1.098 \mathrm{E}-02$ & $-5.239 \mathrm{E}-04$ & 0.9995 \\
\hline MAD & 1.093 & 4.806 & $2.961 \mathrm{E}-03$ & 1.0071 & -0.0986 & $-6.075 \mathrm{E}-03$ & $-2.899 \mathrm{E}-04$ & 0.9997 \\
\hline MADD & 1.079 & 4.868 & $3.271 \mathrm{E}-03$ & 1.0199 & -0.1342 & $-9.016 \mathrm{E}-03$ & $-4.303 \mathrm{E}-04$ & 0.9996 \\
\hline MinMax & 1.055 & 4.909 & $3.466 \mathrm{E}-03$ & 1.0286 & -0.1948 & $-1.375 \mathrm{E}-02$ & $-6.564 \mathrm{E}-04$ & 0.9993 \\
\hline DMinMax & 1.114 & 4.819 & $3.390 \mathrm{E}-03$ & 1.0093 & -0.0238 & $-1.677 \mathrm{E}-03$ & $-8.009 \mathrm{E}-05$ & 0.9999 \\
\hline RMinMax & 0.973 & 4.963 & $9.208 \mathrm{E}-03$ & 1.0243 & -0.1626 & $-3.016 \mathrm{E}-02$ & $-1.462 \mathrm{E}-03$ & 0.9985 \\
\hline RDMinMax & 1.020 & 4.980 & $8.463 \mathrm{E}-03$ & 1.0308 & -0.1209 & $-2.054 \mathrm{E}-02$ & $-9.923 \mathrm{E}-04$ & 0.99900 \\
\hline
\end{tabular}

${ }^{\mathrm{a}}$ Monthly returns and standard deviations in $\%$

${ }^{\mathrm{b}}$ Tracking error measured by standard deviation of portfolio excess return to MSCI ( $\left.\sigma(\mathrm{XB}-\mathrm{Y})\right)$

${ }^{\mathrm{c}}$ Beta of each portfolio to MSCI world stock market index

${ }^{\mathrm{d}}$ Excess return to square root of tracking error in\%

${ }^{\mathrm{e}}$ Excess return to volatility ratio in \%

${ }^{\mathrm{f}}$ Excess return to Beta in \%

${ }^{\mathrm{g}}$ Relative performance of tracking portfolio to benchmark index

Table 7. Comparison of different model returns based on test data

\begin{tabular}{|c|c|c|c|c|c|c|c|}
\hline Date & QTE & MAD & MADD & MinMax & DMinMax & RMinMax & RDMinMax \\
\hline 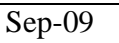 & 0.008594 & 0.004411 & 0.00651 & 0.011516 & -0.00162 & -0.00188 & -0.00245 \\
\hline Oct-09 & 0.009085 & 0.008935 & 0.010766 & 0.010864 & 0.012327 & 0.014258 & 0.01314 \\
\hline Nov-09 & 0.034821 & 0.03549 & 0.036237 & 0.035621 & 0.037809 & 0.035862 & 0.03639 \\
\hline Dec-09 & 0.007785 & 0.006838 & 0.007738 & 0.009098 & 0.006384 & 0.004086 & 0.00370 \\
\hline Jan-10 & -0.00035 & -0.00161 & -0.00083 & 0.00112 & -0.00108 & -0.00664 & -0.00758 \\
\hline Feb-10 & 0.00812 & 0.009915 & 0.00846 & 0.006055 & 0.010439 & 0.013212 & 0.01397 \\
\hline Mar-10 & 0.018063 & 0.020006 & 0.019134 & 0.01693 & 0.023375 & 0.021688 & 0.02186 \\
\hline Apr-10 & 0.006165 & 0.005031 & 0.00462 & 0.006107 & 0.000701 & -0.00178 & -0.00024 \\
\hline Мay-10 & -0.00737 & -0.0081 & -0.00627 & -0.00631 & -0.00776 & 0.004762 & 0.00286 \\
\hline Jun-10 & -0.01299 & -0.01371 & -0.01217 & -0.01161 & -0.01226 & -0.00779 & -0.00899 \\
\hline Jul-10 & -0.00783 & -0.00603 & -0.00708 & -0.00913 & -0.00349 & -0.00449 & -0.00452 \\
\hline Aug-10 & -0.01795 & -0.01892 & -0.01516 & -0.01439 & -0.01401 & -0.00646 & -0.00938 \\
\hline Sep-10 & 0.007881 & 0.00891 & 0.010762 & 0.010106 & 0.01639 & 0.011103 & 0.00968 \\
\hline Oct-10 & 0.000664 & 0.002712 & 0.000661 & -0.00251 & 0.003426 & 0.011006 & 0.01119 \\
\hline Nov-10 & -0.02199 & -0.02138 & -0.01994 & -0.02087 & -0.01689 & -0.01275 & -0.01427 \\
\hline Dec-10 & 0.011974 & 0.010779 & 0.010554 & 0.011132 & 0.007198 & 0.016461 & 0.01553 \\
\hline Jan-11 & -0.00782 & -0.01105 & -0.00638 & -0.00356 & -0.00932 & 0.004923 & 0.00119 \\
\hline Feb-11 & -0.00855 & -0.00833 & -0.00859 & -0.00866 & -0.00861 & -0.0108 & -0.01007 \\
\hline Mar-11 & 0.009302 & 0.009344 & 0.009372 & 0.009542 & 0.009458 & 0.006754 & 0.00712 \\
\hline Apr-11 & -0.00506 & -0.00534 & -0.00543 & -0.006 & -0.00739 & 0.002777 & 0.00309 \\
\hline May-11 & -0.01076 & -0.01102 & -0.00935 & -0.00983 & -0.00726 & 0.002906 & 0.00023 \\
\hline Jun-11 & -0.01558 & -0.01499 & -0.01486 & -0.01562 & -0.01221 & -0.0111 & -0.01224 \\
\hline Jul-11 & -0.00159 & -0.00065 & -0.00026 & -0.0009 & 0.00335 & -0.00212 & -0.00216 \\
\hline Aug-11 & -0.03724 & -0.03428 & -0.03493 & -0.03853 & -0.02709 & -0.02525 & -0.02636 \\
\hline Sep-11 & 0.027016 & 0.031645 & 0.0281 & 0.024189 & 0.036441 & 0.015293 & 0.01988 \\
\hline Oct-11 & -0.00421 & -0.00136 & -0.00187 & -0.00502 & 0.00437 & 0.002724 & 0.00239 \\
\hline Nov-11 & 0.017178 & 0.017599 & 0.017729 & 0.017708 & 0.018574 & 0.012912 & 0.01380 \\
\hline Dec-11 & 0.015416 & 0.014657 & 0.014817 & 0.01526 & 0.012004 & 0.016806 & 0.01753 \\
\hline Jan-12 & -0.00637 & -0.00471 & -0.00334 & -0.00429 & 0.002286 & -0.00812 & -0.00779 \\
\hline Feb-12 & -0.06041 & -0.05847 & -0.05902 & -0.06221 & -0.05332 & -0.04175 & -0.04453 \\
\hline Mar-12 & -0.01886 & -0.023 & -0.01993 & -0.01701 & -0.02648 & -0.00257 & -0.00626 \\
\hline Apr-12 & -0.02094 & -0.02224 & -0.02004 & -0.01907 & -0.02035 & -0.01174 & -0.01468 \\
\hline May-12 & -0.01229 & -0.01317 & -0.01199 & -0.01237 & -0.01309 & 0.003844 & 0.00196 \\
\hline Jun-12 & -0.05353 & -0.05345 & -0.0514 & -0.05457 & -0.05038 & -0.0127 & -0.01644 \\
\hline Jul-12 & 0.002801 & 0.000899 & 0.003529 & 0.004541 & 0.001123 & 0.016893 & 0.01426 \\
\hline Aug-12 & 0.031605 & 0.032491 & 0.030931 & 0.03113 & 0.031967 & 0.016691 & 0.01789 \\
\hline Sep-12 & 0.011932 & 0.012627 & 0.012665 & 0.011807 & 0.014753 & 0.015535 & 0.01446 \\
\hline
\end{tabular}

All figures in \% 
Mohsen Gharakhani et al. / Journal of Computer Science 10 (12): 2450.2463, 2014

Table 8. Tracking error values of noisy data for 50 runs of test data simulation

\begin{tabular}{|c|c|c|c|c|c|c|c|}
\hline Period & QTE & MAD & MADD & MinMax & DMinMax & RMinMax & RDMinMax \\
\hline 1 & 0.0110 & 0.0109 & 0.0109 & 0.0113 & 0.0117 & 0.0141 & 0.0136 \\
\hline 2 & 0.0164 & 0.0164 & 0.0163 & 0.0167 & 0.0164 & 0.0177 & 0.0176 \\
\hline 3 & 0.0209 & 0.0206 & 0.0206 & 0.0213 & 0.0206 & 0.0209 & 0.0206 \\
\hline 4 & 0.0319 & 0.0318 & 0.0317 & 0.0324 & 0.0322 & 0.0316 & 0.0316 \\
\hline 5 & 0.0394 & 0.0395 & 0.0395 & 0.0395 & 0.0397 & 0.0387 & 0.0388 \\
\hline 6 & 0.0317 & 0.0317 & 0.0317 & 0.0318 & 0.0321 & 0.0299 & 0.0300 \\
\hline 7 & 0.0351 & 0.0352 & 0.0353 & 0.0354 & 0.0358 & 0.0314 & 0.0318 \\
\hline 8 & 0.0347 & 0.0349 & 0.0349 & 0.0351 & 0.0363 & 0.0326 & 0.0327 \\
\hline 9 & 0.0373 & 0.0374 & 0.0372 & 0.0380 & 0.0388 & 0.0353 & 0.0354 \\
\hline 10 & 0.0414 & 0.0412 & 0.0410 & 0.0425 & 0.0426 & 0.0382 & 0.0383 \\
\hline 11 & 0.0360 & 0.0356 & 0.0352 & 0.0374 & 0.0377 & 0.0343 & 0.0340 \\
\hline 12 & 0.0394 & 0.0389 & 0.0387 & 0.0406 & 0.0400 & 0.0361 & 0.0359 \\
\hline 13 & 0.0428 & 0.0424 & 0.0424 & 0.0438 & 0.0431 & 0.0381 & 0.0382 \\
\hline 14 & 0.0450 & 0.0447 & 0.0446 & 0.0460 & 0.0456 & 0.0401 & 0.0401 \\
\hline 15 & 0.0430 & 0.0424 & 0.0423 & 0.0442 & 0.0431 & 0.0398 & 0.0395 \\
\hline 16 & 0.0440 & 0.0434 & 0.0433 & 0.0453 & 0.0445 & 0.0397 & 0.0393 \\
\hline 17 & 0.0531 & 0.0521 & 0.0520 & 0.0548 & 0.0517 & 0.0465 & 0.0464 \\
\hline 18 & 0.0528 & 0.0518 & 0.0518 & 0.0545 & 0.0508 & 0.0452 & 0.0450 \\
\hline 19 & 0.0574 & 0.0567 & 0.0570 & 0.0586 & 0.0553 & 0.0469 & 0.0472 \\
\hline 20 & 0.0545 & 0.0539 & 0.0543 & 0.0558 & 0.0529 & 0.0427 & 0.0430 \\
\hline 21 & 0.0566 & 0.0559 & 0.0563 & 0.0579 & 0.0544 & 0.0453 & 0.0458 \\
\hline 22 & 0.0502 & 0.0498 & 0.0501 & 0.0512 & 0.0495 & 0.0416 & 0.0419 \\
\hline 23 & 0.0498 & 0.0498 & 0.0501 & 0.0504 & 0.0499 & 0.0434 & 0.0435 \\
\hline 24 & 0.0493 & 0.0490 & 0.0492 & 0.0500 & 0.0484 & 0.0439 & 0.0438 \\
\hline 25 & 0.0494 & 0.0496 & 0.0500 & 0.0498 & 0.0494 & 0.0433 & 0.0434 \\
\hline 26 & 0.0576 & 0.0580 & 0.0586 & 0.0579 & 0.0579 & 0.0485 & 0.0489 \\
\hline 27 & 0.0579 & 0.0585 & 0.0591 & 0.0579 & 0.0579 & 0.0477 & 0.0482 \\
\hline 28 & 0.0618 & 0.0626 & 0.0633 & 0.0617 & 0.0620 & 0.0493 & 0.0504 \\
\hline 29 & 0.0672 & 0.0681 & 0.0689 & 0.0671 & 0.0670 & 0.0513 & 0.0529 \\
\hline 30 & 0.0668 & 0.0679 & 0.0687 & 0.0663 & 0.0676 & 0.0533 & 0.0548 \\
\hline 31 & 0.0764 & 0.0777 & 0.0787 & 0.0757 & 0.0769 & 0.0607 & 0.0627 \\
\hline 32 & 0.0953 & 0.0967 & 0.0978 & 0.0946 & 0.0956 & 0.0771 & 0.0793 \\
\hline 33 & 0.0993 & 0.1009 & 0.1023 & 0.0987 & 0.0991 & 0.0722 & 0.0755 \\
\hline 34 & 0.0810 & 0.0822 & 0.0832 & 0.0807 & 0.0815 & 0.0612 & 0.0637 \\
\hline 35 & 0.0885 & 0.0901 & 0.0912 & 0.0878 & 0.0893 & 0.0661 & 0.0691 \\
\hline 36 & 0.0952 & 0.0970 & 0.0984 & 0.0944 & 0.0952 & 0.0682 & 0.0718 \\
\hline 37 & 0.1023 & 0.1043 & 0.1059 & 0.1013 & 0.1020 & 0.0700 & 0.0744 \\
\hline 38 & 0.1127 & 0.1143 & 0.1156 & 0.1118 & 0.1124 & 0.0884 & 0.0915 \\
\hline 39 & 0.1069 & 0.1085 & 0.1099 & 0.1062 & 0.1067 & 0.0808 & 0.0840 \\
\hline 40 & 0.1093 & 0.1111 & 0.1127 & 0.1085 & 0.1089 & 0.0788 & 0.0824 \\
\hline 41 & 0.1183 & 0.1200 & 0.1215 & 0.1176 & 0.1179 & 0.0884 & 0.0920 \\
\hline 42 & 0.1047 & 0.1066 & 0.1083 & 0.1041 & 0.1044 & 0.0698 & 0.0742 \\
\hline 43 & 0.1211 & 0.1231 & 0.1252 & 0.1205 & 0.1197 & 0.0777 & 0.0831 \\
\hline 44 & 0.1101 & 0.1114 & 0.1127 & 0.1097 & 0.1097 & 0.0853 & 0.0881 \\
\hline 45 & 0.1184 & 0.1198 & 0.1212 & 0.1180 & 0.1177 & 0.0902 & 0.0934 \\
\hline 46 & 0.1345 & 0.1361 & 0.1376 & 0.1340 & 0.1340 & 0.1061 & 0.1093 \\
\hline 47 & 0.1410 & 0.1428 & 0.1446 & 0.1404 & 0.1403 & 0.1052 & 0.1093 \\
\hline 48 & 0.1565 & 0.1590 & 0.1616 & 0.1557 & 0.1548 & 0.1043 & 0.1105 \\
\hline 49 & 0.1917 & 0.1946 & 0.1975 & 0.1907 & 0.1900 & 0.1358 & 0.1423 \\
\hline 50 & 0.1486 & 0.1510 & 0.1533 & 0.1479 & 0.1472 & 0.1010 & 0.1067 \\
\hline
\end{tabular}


Mohsen Gharakhani et al. / Journal of Computer Science 10 (12): 2450.2463, 2014

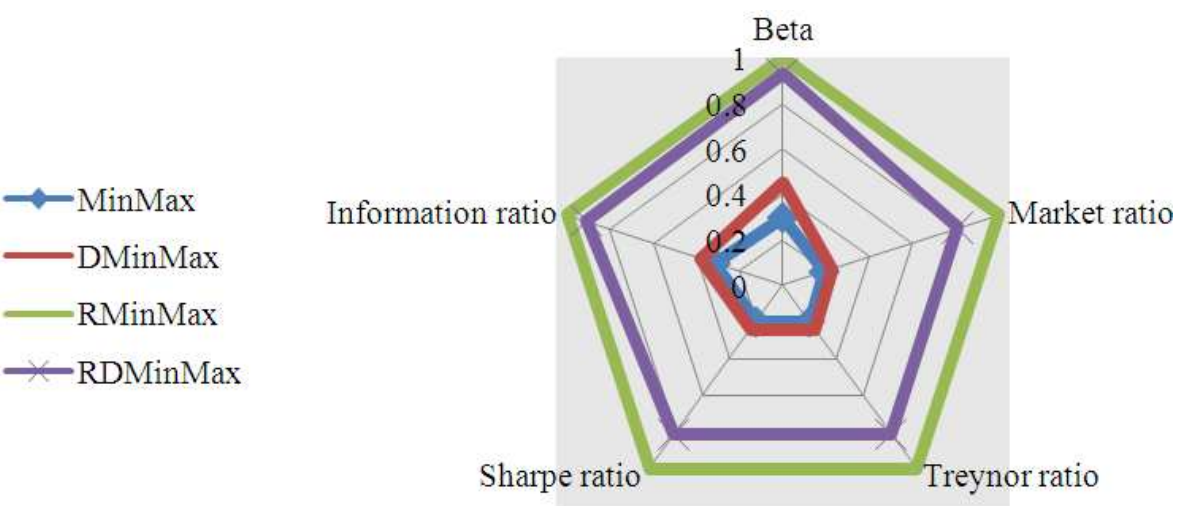

Fig. 1. Comparison of different models based on train period return

Comparing different robust models

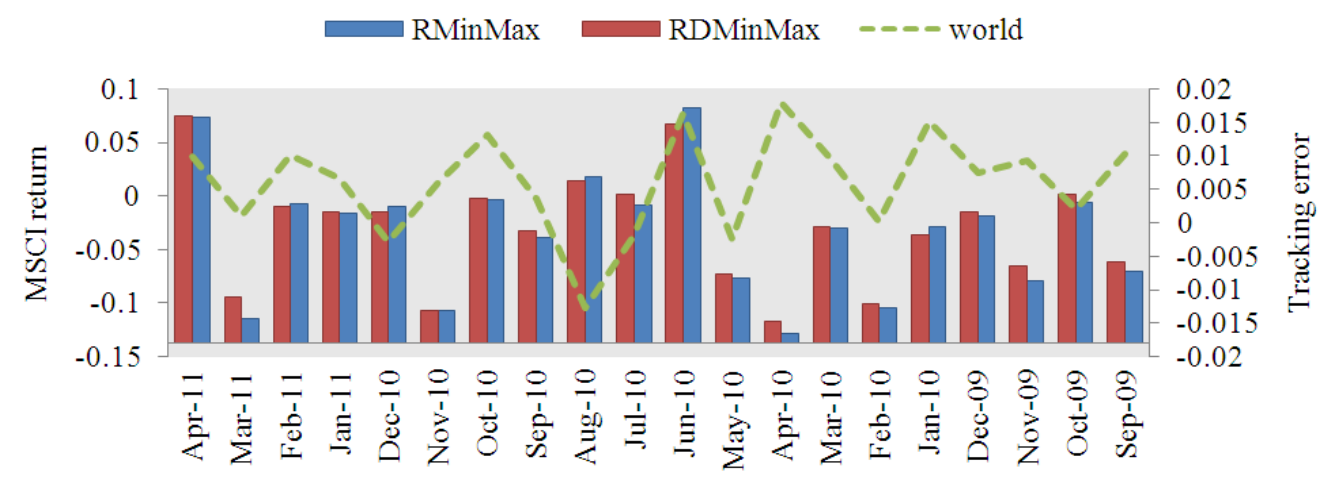

Out of the sample dates

Fig. 2. The comparison of the tracking error of the robust models regarding the world index fluctuations

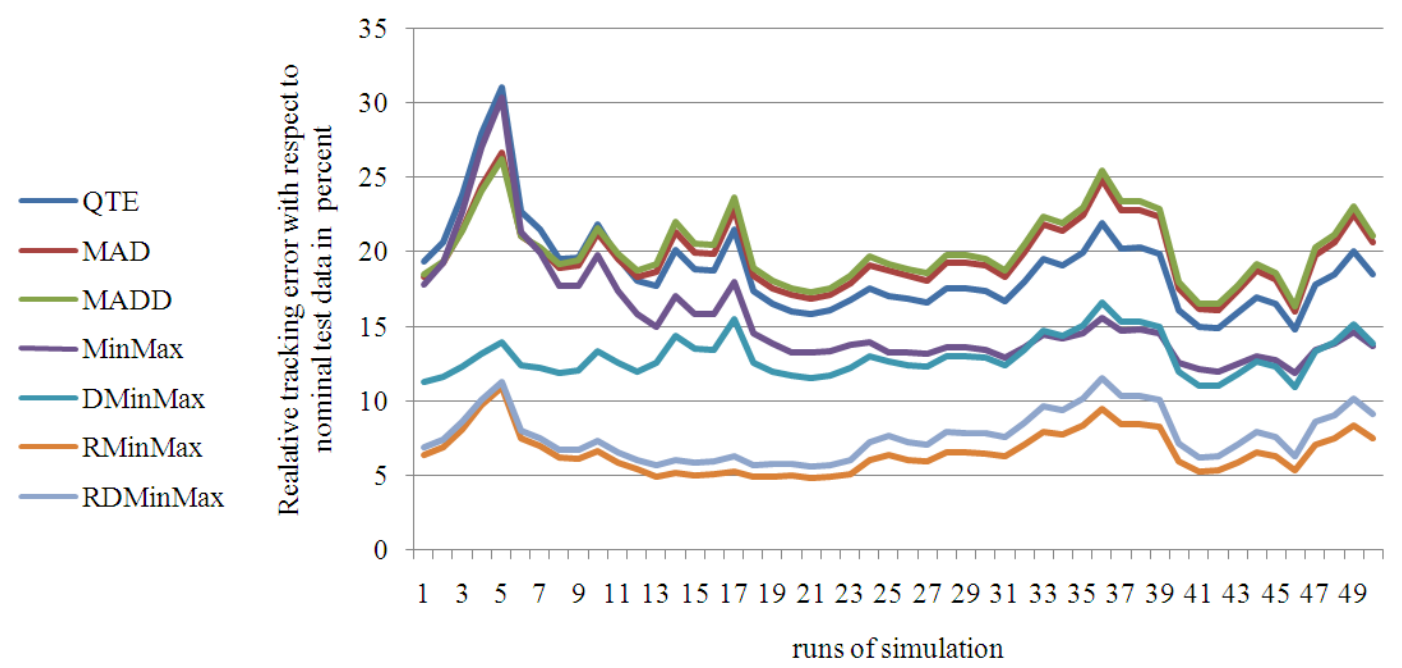

Fig. 3. Comparison of relative tracking error of different models for noisy data with respect to nominal test data for 50 runs of simulation (in percent) 


\section{CONCLUSION}

In this study, we presented some linear programming formulations for index tracking and their robust counterparts. We proposed a robust modeling approach for index tracking and compared it with the well-known mean square error and other linear models in the literature. The performance of the proposed models is analyzed using a real-world data set including six national stock market indices and their tracking error is minimized with respect to world stock market index MSCI. The results of the comparison based on train data, reveal that while the standard deviation of returns are almost the same, the proposed robust formulations outperform different portfolios regarding the mean return. The comparison using nominal test data shows that the tracking error in robust models has been increased, although the returns and standard deviations still remained the same. In the next stage, we added a random noise to the test data that has normal distribution and has run the simulation for 50 times. This time the tracking error in robust models was relatively less than the non-robust models. This proves better performance of robust models when data are noisy. In addition, relative tracking error for noisy data with respect to nominal data was compared among the models. The results suggest that relative tracking error changes for the robust models were less than the non-robust models.

In conclusion it was revealed that portfolio weights of the optimized portfolio and their corresponding return over their risk profile are different across the models. This difference implies that optimization models should be targeted to a specific investment attitude. Nonetheless, this new proposed model of portfolio selection problems and the efficient solution methods will allow us to solve more complicated problems in real world situations under more random and ambiguous conditions. For future research, we suggest applying this robust portfolio selection approach to the other asset allocation problems including multi-period planning horizon, cardinality constraint, transaction costs and portfolio insurance.

\section{REFERENCES}

Ammann, M. and H. Zimmermann, 2001. Tracking error and tactical asset allocation. Financial Anal. J., 57: 32-43. DOI: 10.2469/faj.v57.n2.2431

Ben-Tal, A. and A. Nemirovski, 1998. Robust convex optimization. Math. Operat. Res., 23: 769-805. DOI: 10.1287/moor.23.4.769
Ben-Tal, A. and A. Nemirovski, 1999. Robust solutions of uncertain linear programs. Operat. Res. Lett., 25: 1-14. DOI: 10.1016/S0167-6377(99)00016-4

Ben-Tal, A. and A. Nemirovski, 2000. Robust solutions of linear programming problems contaminated with uncertain data. Math. Programm., 88: 411-424. DOI: 10.1007/PL00011380

Ben-Tal, A. and A. Nemirovski, 2002. Robust optimizationmethodology and applications. Math. Programm., 92: 453-480. DOI: 10.1007/s101070100286

Bertrand, P., 2010. Another look at portfolio optimization under tracking-error constraints. Financial Anal. J., 66: 78-90. DOI: 10.2469/faj.v66.n3.2

Bertsimas, D. and M. Sim, 2004. The price of robustness. Operat. Res., 52: 35-53. DOI: 10.1287/opre.1030.0065

Chen, C. and R.H. Kwon, 2012. Robust portfolio selection for index tracking. Comput. Operat. Res., 3: 829-837. DOI: 10.1016/j.cor.2010.08.019

Cornuejols, G. and R. Tütüncü, 2007. Optimization methods in finance. Cambridge University Press, New York. ISBN-10: 9780521861700

Derigs, U. and N.H. Nickel, 2003. Meta-heuristic based decision support for portfolio optimization with a case study on tracking error minimization in passive portfolio management. OR Spectrum, 25: 345-378. DOI: $10.1007 / \mathrm{s} 00291-003-0127-5$

Doan, X., X. Li and K. Natarajan, 2013. Robustness to dependency in portfolio optimization using overlapping marginals. Techn. Report. NUS. DOI: DB_FILE/2013/05/3876

El-Ghaoui, L., F. Oustry and H. Lebret, 1998. Robust solutions to uncertain semidefinite programs. SIAM J. Optimizat., 9: 33-52. DOI: 10.1137/S1052623496305717

El Ghaoui, L., M. Oks and F. Oustry, 2003. Worst-case value-at-risk and robust portfolio optimization: A conic programming approach. Operat. Res., 54: 543556. DOI: 10.1287/opre.51.4.543.16101

Fabozzi, F.J., 2007. Robust portfolio optimization and management. Wiley, New Jersey. ISBN: 978-0471-92122-6

Fabozzi, F.J., S.M. Focardi and C.L. Jonas, 2004. Trends in quantitative asset, management in Europe. J. Portfolio Manag., 31: 125-132. DOI: 10.3905/jpm.2004.125

Fertis, A., M. Baes and H.J. Luthi, 2012. Robust risk management. Eur. J. Operat. Res., 222: 663-672. DOI: 10.1016/j.ejor.2012.03.036 
Gabrel, V., C. Murat and A. Thiele, 2013. Recent Advances in Robust Optimization: An Overview. Eur. J. Operat. Res., 235: 471-483. DOI: 10.1016/j.ejor.2013.09.036

Gaivoronski, A.A. and G. Pflug, 2005. Value at risk in portfolio optimization: Properties and computational approach. J. Risk, 7: 1-31. DOI: 10.1.1.27.1398

Goldfarb, D. and G. Iyengar, 2003. Robust portfolio selection problems. Math. Operat. Res., 28: 1-38. DOI: $10.1287 /$ moor.28.1.1.14260

Gregory, C., K. Darby-Dowman and G. Mitra, 2011. Robust optimization and portfolio selection: The cost of robustness. Eur. J. Operat. Res., 212: 417428. DOI: 10.1016/j.ejor.2011.02.015

Harlow, W.V., 1991. Asset allocation in a downside-risk framework. Financial Anal. J., 47: 28-40. DOI: 10.2469/faj.v47.n5.28

Jansen, R. and R. Van Dijk, 2002. Optimal benchmark tracking with small portfolios. J. Portfolio Manag., 28: 33-39. DOI: 10.3905/jpm.2002.319830

Jorion, P., 2003. Portfolio optimization with trackingerror constraints. Financial Anal. J., 59: 70-82. DOI: 10.2469/faj.v59.n5.2565

Kawas, B. and A. Thiele, 2011. A log-robust optimization approach to portfolio management. OR Spectrum, 33: 207-233. DOI: 10.1007/s00291-008-0162-3

Konno, H. and H. Yamazaki, 1991. Mean-absolute deviation portfolio optimization model and its applications to Tokyo stock market. Manag. Sci., 37: 519-531. DOI: 10.1287/mnsc.37.5.519

Konno, H., K. Akishino and R. Yamamoto, 2005. Optimization of a long-short portfolio under no convex transaction cost. Comput. Optimizat. Applic., 32: 115-132. DOI: $10.1007 / \mathrm{s} 10589-005-2056-5$

Lai, K.K., L. Yu and S. Wang, 2006. Mean-varianceskewness-kurtosis-based portfolio optimization. Comput. Comput. Sci., 2: 292-297. DOI: 10.1109/IMSCCS.2006.239
Markowitz, H., 1952. Portfolio selection. J. Finance, 7: 77-91. DOI: 10.1111/j.1540-6261.1952.tb01525.x

Markowitz, H., 1952. Portfolio selection. J. Finance, 7: 77-91. DOI: 10.1111/j.1540-6261.1952.tb01525.x

Meade, N. and G.R. Salkin, 1990. Developing and maintaining an equity index fund. J. Operat. Res. Soc., 41: 599-607. DOI: 10.1057/jors.1990.84

Mulvey, J.M., R.J. Vanderbei and S.A. Zenios, 1995. Robust optimization of large-scale systems. Operat. Res., 43: 264-281. DOI: 10.1287/opre.43.2.264

Nguyen, T. and A. Lo, 2012. Robust ranking and portfolio optimization. Eur. J. Operat. Res., 221: 407-416. DOI: 10.1016/j.ejor.2012.03.023

Pinar, M.C. and R.H. Tutuncu, 2005. Robust profit opportunities in risky financial portfolios. Operat. Res. Lett., 33: 331-340. DOI: 10.1016/j.orl.2004.08.005

Roll, R., 1992. A mean/variance analysis of tracking error. J. Portfolio Manag., 18: 13-22. DOI: 10.3905/jpm.1992.701922

Rudolf, M., H.J. Wolter and H. Zimmermann, 1999. A linear model for tracking error minimization. J. Banking Finance, 23: 85-103. DOI: 10.1016/S03784266(98)00076-4

Sharpe, W.F., 1966. Mutual Fund Performance. J. Bus., 39: $119-138$.

Sharpe, W.F., 1994. The sharpe ratio. J. Portfolio Manag., 21: 49-58. DOI: 10.3905/jpm.1994.409501

Soyester, A.L., 1973. Convex programming with setinclusive constraints and applications to inexact linear programming. Operat. Res., 21: 1154-1157. DOI: $10.1287 /$ opre.21.5.1154

Treynor, J.L., 1964. How to rate management of investment funds. Harvard Bus. Rev., 43: 63-75.

Yao, D.D., S. Zhang and X.Y. Zhou, 2006. Tracking a financial benchmark using a few assets. Operat. Res., 54: 232-246. DOI: 10.1287/opre.1050.0260

Zhu, S., M. Fan and D. Li, 2013. Portfolio management with dual robustness in prediction and optimization: A mixture model based learning approach. Social Sci. Res. Netw., DOI: 10.2139/ssrn.2320292 